\section{Dementia Screening Questionnaire for Individuals with Intellectual Disabilities}

\author{
SHOUMITRO DEB, MONIKA HARE, LINDSAY PRIOR \\ and SABYASACHI BHAUMIK
}

\begin{abstract}
Background Many adults with Down's syndrome develop Alzheimer's dementia relatively early in their lives, but accurate clinical diagnosis remains difficult.
\end{abstract}

\begin{abstract}
Aims To develop a user-friendly observer-rated dementia screening questionnaire with strong psychometric properties for adults with intellectual disabilities.
\end{abstract}

Method We used qualitative methods to gather information from carers of people with Down's syndrome about the symptoms of dementia. This provided the items for the Dementia Screening Questionnaire for Individuals with Intellectual Disabilities (DSQIID), which we then tested for its psychometric properties.

Results The DSQIID was administered to carers of 193 adults with Down's syndrome, 117 of whom were examined by clinicians who confirmed a diagnosis of dementia for 49 according to modified ICD-10 criteria. We established that a total score of 20 provides maximum sensitivity (0.92) and optimum specificity (0.97) for screening. The DSQIID has sound internal consistency ( $\alpha=0.91)$ for all its 53 items, and good test-retest and interrater reliability. We established a good construct validity by dividing the items into four factors.

\section{Conclusions The DSQIID is a valid, reliable and user-friendly observer-rated questionnaire for screening for dementia among adults with Down's syndrome.}

Declaration of interest None. Funding detailed in Acknowledgements.
Alzheimer's dementia is relatively common among adults with Down's syndrome and tends to manifest relatively early. As in the general population, increasing age and genetic predisposition act as risk factors (Aylward et al, 1997; Deb et al, 2000). Autopsy and neuroimaging studies (Deb et al, 1992) have shown an almost universal presence of Alzheimer's neuropathology among adults with Down's syndrome over the age of 45 years. Clinically, however, dementia is not universally manifested in this population (Mann, 1988; Prasher, 1995). One of the reasons for this discrepancy is the difficulty in diagnosing dementia among people with Down's syndrome in particular, and intellectual disabilities in general - especially during the early stage of dementia. Unfortunately, screening methods used for the detection of dementia among the general population are not suitable for people with intellectual disabilities because of floor effects. Moreover, we cannot standardise the cut-off thresholds for people with intellectual disabilities because those people vary considerably in their cognitive abilities. For the same reasons direct neuropsychological tests, including the Mini-Mental State Examination (MMSE; Folstein et al, 1975), are not useful for this population. Therefore, an observer-rated screening instrument which is primarily based on the reporting of behavioural changes following the onset of dementia is desirable (Deb \& Braganza, 1999).

The two dementia screening instruments that are currently in wide use among people with intellectual disabilities, namely the Dementia Scale for Down Syndrome (DSDS; Gedye, 1995) and the Dementia Questionnaire for Persons with Mental Retardation (DMR; Evenhuis, 1992, 1996), both have drawbacks. A questionnaire that is valid, reliable and easy to use could help to screen for dementia among people with Down's syndrome, which will help in timely diagnosis and treatment. We therefore developed a behavioural rating scale, incorporating carers' perspectives at the outset, for use by carers to screen for dementia in people with intellectual disabilities.

\section{METHOD}

\section{Questionnaire development}

We followed the steps described by Streiner \& Norman (1999), which are widely accepted as gold standards for developing a new questionnaire. A qualitative interview method was used to inform the development of the questionnaire. Data gathered from interviews with carers of 24 adults with Down's syndrome and dementia were analysed qualitatively to derive 53 items for inclusion in the new questionnaire. The age of the 24 adults with Down's syndrome ranged from 48 to 72 years. Four people had mild, 16 moderate and 4 severe intellectual disabilities according to the ICD-10 criteria (World Health Organization, 1992). We named the questionnaire the Dementia Screening Questionnaire for Individuals with Intellectual Disabilities (DSQIID) because although the questionnaire was only tested among adults with Down's syndrome, we believe that it can be used equally effectively among all adults with intellectual disabilities. The project received approval from the Welsh Multicentre Research Ethics Committee and we obtained written consent from each carer who completed the questionnaire.

The DSQIID is an observer-rated questionnaire, which is completed by carers of people with Down's syndrome who have known the individual for some time. The DSQIID is divided into three parts (see data supplement to online version of this paper). The first asks about the 'best' ability the person has or has had. The second contains 43 questions about behaviour or symptoms that are usually associated with dementia in adults with Down's syndrome. Each item is scored on a four-point scale: 'always has been the case'; 'always, but worse'; 'new symptoms'; and 'does not apply'. We adopted this scoring system to overcome the floor effect of the existing dementia screening scales, which only score current behaviour and not changes in behaviour (because in the general population the preexistence of these skills is presumed). Items with a response of 'always been the case' or 'does not apply' are scored 0 , those with 'always but worse' or 'new symptom' are scored 1.

Part 3 of the DSQIID contains 10 questions, all of which are comparative; for 
example, 'speaks (signs) less' and 'seems generally more tired'. A response of 'yes' is scored 1 and a response of 'no' is scored 0 . Scores from parts 2 and 3 are added to provide a total score. The 53 items of the DSQIID cover areas such as loss of memory, confusion, loss of skills, social withdrawal, behavioural changes, psychological symptoms, physical symptoms, sleep disturbance and speech abnormalities.

\section{Questionnaire evaluation}

\section{Sample selection}

Initially S.D. contacted colleagues in the UK requesting them to identify adults with Down's syndrome with and without dementia who might be suitable for inclusion in the study. M.H. publicised the study among her contacts who are primarily carers in Wales. S.B. approached those carers of adults with Down's syndrome on the Leicestershire register who had agreed to take part in research. The Leicestershire register holds information on over 3000 people with intellectual disabilities in the county. The adults with Down's syndrome who were included in the study had a range of intellectual disabilities.

Carers who expressed an interest were sent an information sheet, a written consent form, the DSQIID and a stamped addressed envelope. They were asked to return the completed DSQIID along with the completed consent form. We also asked the first carer to inform us of any other carer of the person with Down's syndrome who was willing to complete a DSQIID for that person - this was done to assess interrater reliability. Where appropriate, we immediately sent the same pack to the second carer, and thereby managed to gather data from 41 second carers of adults with Down's syndrome. We also sent the DSQIID again to the same carers immediately after we had received their completed first questionnaire. By this means we gathered test-retest data for 52 adults with Down's syndrome.

\section{Inclusion criteria and matching}

We did not match the groups with and without dementia for age and gender but subsequent analysis showed that those with dementia were significantly older than those without, which was expected. There was no significant difference in gender distribution between the groups. We did not match the two groups according to other possible confounders, such as hypothyroidism and depression, but on subsequent data analysis we did not find any significant intergroup differences in these variables (see Table 1).

\section{Data analysis}

We entered all data anonymously and analysed them using SPSS version 13 for Windows (Field, 2005).

\section{RESULTS}

\section{Demographic data}

We gathered data using the DSQIID on 193 adults with Down's syndrome from 28 centres in the UK. Local clinicians examined 117 of these adults and confirmed a diagnosis of dementia among 49 and the absence of dementia among 68 according to the modified ICD-10 criteria for the diagnosis of dementia among adults with intellectual disabilities (Aylward et al, 1997). Because some adults with Down's syndrome were recruited through carers and nursing staff, 76 were not examined by a clinician and therefore we do not have a dementia diagnosis for these participants. We used receiver operating characteristic (ROC) analysis only on data from those who were examined by a clinician. We excluded 1 person with Down's syndrome from the ROC analysis because he had a mixed diagnosis of cerebrovascular events and dementia. We used data from all participants to analyse test-retest and interrater reliability.

The age of the whole cohort ranged from 23 to 77 years (mean 55 years, s.d.=7.6); $51 \%$ were male. The age of the 49 adults with dementia ranged from 44 to 77 years (mean 56 years, s.d.=7). The age range of 68 adults without dementia was 23-63 years (mean 44 years, s.d.=10). Eighteen adults without dementia were over age 50 . Independent-sample $t$ test showed that those with dementia were significantly older than those without $(P<0.001) ; 54 \%$ of those with dementia and $37 \%$ of those without were female. Although it was not possible to gather IQ scores from a cohort recruited from multiple centres, $35 \%$ had fluent speech, $37 \%$ could use short sentences, $15 \%$ speak a few words, $7 \%$ used sign language and $6 \%$ had no speech. Similarly, $13 \%$ lived totally independently, $6 \%$ lived independently but needed a lot of help, $35 \%$ were cared for by others and needed some help, and $46 \%$ were cared for by others and needed a lot of help for self-care. Therefore, it could be assumed that a proportion had severe and profound intellectual disabilities.

Comparative data for the adults with and without a diagnosis of dementia on the presence of depression, epilepsy, visual or hearing problems, and the use of anti-epileptics, antidepressants and thyroxine are presented in Table 1. A significantly higher proportion of adults with dementia had hearing $(P=0.014)$ and visual $(P=0.044)$ problems.

\section{Psychometric properties}

\section{Feasibility}

We sought comments from experts on the initial draft, and updated the questionnaire in the light of comments received. We piloted the draft questionnaire among six carers of adults with Down's syndrome and dementia to identify any practical difficulties before wider use in field-testing. Any ambiguity in the questions, difficulty in understanding wording and other practical issues related to the design of the DSQIID were rectified.

\section{Content validity}

We checked whether carers were consistently missing any particular item or providing the same answer. We also checked

Table I Variables in adults with Down's syndrome with $(n=49)$ and without $(n=68)$ a clinical diagnosis of dementia

\begin{tabular}{lcc}
\hline Variable & With dementia $(n=49)$ & Without dementia $(n=68)$ \\
& $n(\%)$ & $n(\%)$ \\
\hline Thyroxine treatment & $12(24.5)$ & $26(40.6)$ \\
Depression & $5(11.4)$ & $3(5)$ \\
Antidepressant treatment & $9(18)$ & $7(11)$ \\
Epilepsy & $13(26.5)$ & $6(10.3)$ \\
Anti-epileptic treatment & $17(33.3)$ & $9(14.1)$ \\
Visual problems & $23(46.9)$ & $18(28.6)^{*}$ \\
Hearing problems & $19(38.8)$ & $12(19)^{* *}$ \\
\hline
\end{tabular}

$* P=0.044 ; * P=0.014$ 
Table 2 Factor analysis of the 43 DSQIID items.

\begin{tabular}{|c|c|c|c|c|}
\hline & $\begin{array}{l}\text { Memory/ } \\
\text { confusion }\end{array}$ & $\begin{array}{l}\text { Feelings of } \\
\text { insecurity }\end{array}$ & $\begin{array}{c}\text { Sleep } \\
\text { problems }\end{array}$ & $\begin{array}{l}\text { Behaviour } \\
\text { problems }\end{array}$ \\
\hline Initial eigenvalues & 17.71 & 3.0 & 2.12 & 2.1 \\
\hline$\%$ Variance & 41.17 & 6.93 & 4.93 & 4.76 \\
\hline 0I Can't wash/ bathe without help & 0.690 & & & \\
\hline 03 Dresses inappropriately & 0.579 & & & \\
\hline 09 Can't find words & 0.678 & & & \\
\hline 10 Can't follow simple instructions & 0.725 & & & \\
\hline II Can't follow more than one instruction & 0.733 & & & \\
\hline 12 Stops in the middle of a task & 0.596 & & & \\
\hline I3 Can't read & 0.433 & & & \\
\hline 14 Can't write & 0.538 & & & \\
\hline 17 Confused at night & 0.603 & & & \\
\hline 20 Can't find way in familiar surroundings & 0.564 & & & \\
\hline 22 Loses track of time & 0.640 & & & \\
\hline 23 Not confident to walk over small cracks & 0.473 & 0.406 & & \\
\hline 26 Can't recognise familiar persons & 0.536 & & & \\
\hline 27 Can't remember names of persons & 0.688 & & & \\
\hline 28 Can't remember recent events & 0.740 & & & \\
\hline 32 Seems to go into own world & 0.554 & & & \\
\hline 37 Does not know what to do with objects & 0.517 & & & \\
\hline 43 Talks to self & 0.541 & & & \\
\hline 02 Can't dress without help & 0.506 & 0.531 & & \\
\hline 05 Needs help eating & & 0.590 & & \\
\hline 06 Needs help using bathroom & & 0.577 & & \\
\hline 07 Incontinence including accidents & 0.412 & 0.420 & & \\
\hline 08 Does not initiate conversation & & 0.537 & & \\
\hline 24 Unsteady walk/loses balance & & 0.531 & & \\
\hline 25 Can't walk unaided & & 0.472 & & \\
\hline 29 Withdraws from social activities & & 0.606 & & \\
\hline 30 Withdraws from persons & & 0.427 & & \\
\hline 3I Loss of interest in hobbies/ activities & & 0.688 & & \\
\hline 38 Appears insecure & & 0.550 & & \\
\hline 39 Appears anxious or nervous & & 0.610 & & \\
\hline 40 Appears depressed & & 0.612 & & \\
\hline 04 Undresses inappropriately & & & 0.492 & \\
\hline 15 Changed sleep pattern & & & 0.672 & \\
\hline 16 Wakes at night & & & 0.674 & \\
\hline 18 Sleeps during the day & & & 0.511 & \\
\hline 19 Wanders at night & & & 0.741 & \\
\hline 2I Wanders & & & 0.711 & \\
\hline 42 Fits/ Epilepsy & & & 0.508 & \\
\hline 33 Obsessive or repetitive behaviour & & & & 0.737 \\
\hline 34 Hides or hoards objects & & & & 0.755 \\
\hline 35 Loses objects & 0.544 & & & 0.581 \\
\hline 36 Puts familiar things into wrong places & 0.525 & & & 0.527 \\
\hline 4I Shows aggression & & & & 0.715 \\
\hline
\end{tabular}

DSQIID, Dementia Screening Questionnaire for Individuals with Intellectual Disabilities.

for possible floor or ceiling effects from the spread of overall scores from all carers. When preparing the questions we took into positive and negative wording, and the length of items.

\section{Construct validity}

An initial principal component analysis using 'varimax rotation' created 13 factors (Field, 2005), which captured about $80 \%$ of the total variance. Subsequent scree plot analysis revealed that between four and five factors would be more appropriate but clinical grouping of items revealed that a four-factor structure was most appropriate for the DSQIID. Therefore, we carried out a forced four-factor analysis with four factors which included over $57 \%$ of the overall variance (Table 2). We excluded from the factor analysis the last 10 items of the DSQIID that were rated on a two-point scale as either 'yes' or 'no' as opposed to other items that were rated on a four-point scoring system (see data supplement to online version of this paper).

Factor 1 has most items involving symptoms of memory deficit and confusion; factor 2 includes primarily symptoms relating to frontal lobe dysfunction such as apathy and feelings of insecurity; factor 3 comprises primarily sleep and confusionrelated items; and factor 4 symptoms associated with behavioural problems. Apart from some minor overlap, the factors appear to reflect different clinical symptoms. About $41 \%$ of the variance is owing to factor 1 , whereas the remaining three factors contribute less than $17 \%$ of the variance. The first new variable contains the maximum amount of variation, whereas the remaining variables are orthogonal to the first and are independent of the first principal component. It is for this reason that the latter three factors contribute less to the variance as any common associations with first component items are ignored. This means that the items comprising factor 1 , such as memory impairment and confusion, are only somewhat more important in screening for dementia in this population than the items in other factors.

\section{Internal consistency \\ Cronbach's $\alpha$ for all 53 items in the DSQIID is 0.91 .}

\section{Criterion-related validity}

We assessed criterion-related validity by comparing the total score on the DSQIID with the clinicians' diagnosis of the presence or absence of dementia among 117 
adults with Down's syndrome. We used a ROC method to calculate the best fit between specificity and sensitivity. Out of 49 adults who had a clinical diagnosis of dementia, 4 scored less than 20 on the DSQIID. Out of 68 adults with Down's syndrome who did not have a clinical diagnosis of dementia, 2 scored more than 20 on the DSQIID. Therefore, use of an overall score of 20 as a screening cut-off provided a specificity of 0.97 , a sensitivity of 0.92 , a positive likelihood ratio of 31 and a negative likelihood ratio of 0.08 . Hence with a cut-off score of 20 a positive diagnosis of dementia is $\mathbf{3 1}$ times more likely in a person with dementia than in one without. Similarly, a negative diagnosis of dementia is 0.08 times more likely or 13 times less likely in a person with dementia than without. We therefore recommend 20 as the cut-off for the total score when using the DSQIID for screening for dementia among adults with Down's syndrome. However, it is possible that there might be a different cut-off score for people with severe and profound intellectual disabilities whom we were unable to test separately. We therefore recommend the serial use of DSQIID over a period of time, particularly for people with severe and profound intellectual disabilities.

\section{Reliability}

The intraclass correlation for test-retest reliability $(n=52)$ is 0.95 , with a two-tailed level of significance of $P<0.01 \quad(>80 \%$ power). The intraclass correlation for interrater reliability $(n=41)$ is 0.9 , and the twotailed level of significance is $P<0.01$ ( $>80 \%$ power).

\section{DISCUSSION}

\section{Development of DSQIID}

Our approach in developing the DSQIID is somewhat unique in that, for the first time, we have adopted a 'bottom up' approach and incorporated the views of carers of adults with Down's syndrome regarding the symptoms of dementia. The strategy has ensured that the DSQIID has good face and content validity. It also puts carers' views, to the forefront.

The DSQIID is easy to use, takes approximately $10-15 \mathrm{~min}$ to complete and can be completed either at home or in a clinic. The questions are simple and easy to understand, and the scoring system is simple and unambiguous. The screening cut-off is constant rather than variable,

SHOUMITRO DEB, MBBS, FRCPsych, MD, University of Birmingham, Division of Neuroscience, Department of Psychiatry, Queen Elizabeth Psychiatric Hospital, Birmingham; MONIKA HARE, MPhil, Welsh Centre for Learning Disabilities, Cardiff; LINDSAY PRIOR, PhD, Department of Sociology, Queen's University, Belfast; SABYASACHI BHAUMIK, MBBS, FRCPsych, Department of Psychiatry, University of Leicester, Leicester, UK.

Correspondence: Professor Shoumitro Deb, Division of Neuroscience, Department of Psychiatry, Queen Elizabeth Psychiatric Hospital, Mindelsohn Way, Birmingham BI5 2QZ,UK. Email: s.deb@bham.ac.uk

(First received 3 April 2006, final revision 27 November 2006, accepted I2 December 2006)

unlike the DSDS. The same cut-off score applies to adults with all levels of intellectual disabilities, unlike the DMR. We were not able to gather data on the level of intellectual disabilities among the whole study population but have included adults with all degrees of intellectual disabilities. Moreover, the original qualitative study used for the design of the DSQIID included adults with Down's syndrome with mild, moderate and severe intellectual disabilities (Deb et al, 2007). We have found that adults with Down's syndrome and severe intellectual disabilities showed a different manifestation of dementia in the early stage of the disease (primarily loss of skills) compared with those with mild-to-moderate intellectual disabilities (primarily memory deficit) (Deb et al, 2007). However, the end-stage symptoms of dementia are likely to be similar in both groups.

\section{Other findings}

Although we did not match those with and without dementia at the outset of the study, data analysis showed that, as expected, the dementia group was significantly older. The gender distribution of the two groups is similar. The two most common differential diagnoses of dementia are depression and hypothyroidism but there were no significant differences between the two groups on these variables. However, both hypothyroidism and depression may coexist with dementia and both are treatable conditions. There was a higher rate of hearing and visual problems among the group with dementia but the implication of these findings is not clear. Perhaps those with sensory deficits are more likely to develop dementia or this group might have been erroneously diagnosed because of their poor sensory skills.

We believe that for the DSQIID to be most effective the carers completing it should have known the person with intellectual disabilities for at least 6 months, and should have witnessed the change in behaviour since before the onset of dementia. Although we did not use this criterion for the field-testing, we believe that the carer should report only those behaviours that have existed for at least 6 months.

\section{Limitations}

It was not possible to test whether a different cut-off score for screening dementia should be applied for people with severe and profound intellectual disabilities. Moreover, sensitivity of the DSQIID can only be tested in a prospective study.

Inclusion of more adults with a clinical diagnosis of dementia might have improved the accuracy of the results.

\section{Strengths}

In order to avoid the floor effect, as can be seen with the MMSE, we have employed a scoring system by which only recent changes in behaviour are scored rather than all behaviours. This is a major strength of the DSQIID, which allows its use in a cross-sectional context. However, it is probably best to use the DSQIID at regular intervals over a period of time to identify the change in score. A further strength of the DSQIID relates to its robust psychometric properties, which existing scales often do not possess. Previous studies have included only a small number of people with dementia when validating scales (Evenhuis, 1992; Gedye, 1995), whereas in this study the number of people with dementia is much higher, and is very close to the 50 suggested by Streiner \& Norman (1999). Moreover, the scores for test-retest and interrater reliability and internal consistency indicate that the DSQIID is very robust compared with existing scales.

\section{ACKNOWLEDGEMENTS}

We thank all the carers who took part in the study and the people with Down's syndrome for whom they cared. We thank Dr Sayeed Haque for statistical advice, staff at the Leicestershire Register for People with Learning Disabilities and clinicians who 
helped with recruitment and examined people with Down's syndrome for the diagnosis of dementia. The study was funded by the Baily Thomas Charitable Trust.

\section{REFERENCES}

Aylward, E. H., Burt, D. B., Thorpe, L. U., et al (1997) Diagnosis of dementia in individuals with intellectual disability. Journal of Intellectual Disability Research, 4I, 152-164.

Deb, S. \& Braganza, J. (1999) Comparison of rating scales for the diagnosis of dementia in adults with Down's syndrome. Journal of Intellectual Disability Research, 43, 400-407.

Deb, S., Braganza, J., Norton, N., et al (2000) APOE $€ 4$ influences the manifestation of Alzheimer's disease in adults with Down's syndrome. British Journal of

Psychiatry, 176, 468-472.
Deb, S., de Silva, P. N., Gemmell, H. G., et al (1992) Alzheimer's disease in adults with Down's syndrome: the relationship between regional blood flow deficits and dementia. Acta Psychiatrica Scandinavica, 86, 340-345.

Deb, S., Hare, M. \& Prior, L. (2007) Symptoms of dementia among adults with Down's syndrome: a qualitative study. Journal of Intellectual Disability Research, in press.

Evenhuis, H. M. (1992) Evaluation of a screening instrument for dementia in ageing mentally retarded persons. Journal of Intellectual Disability Research 36, 337-447.

Evenhuis, H. M. (1996) Further evaluation of the Dementia Questionnaire for Persons with Mental Retardation. Journal of Intellectual Disability Research, 40, 369-373.

Field, A. (2005) Discovering Statistics using SPSS for Windows. Sage Publications.
Folstein, M. F., Folstein, S. E. \& McHugh, P. R. (1975) 'Mini-Mental state'. A practical method for grading the cognitive state of patients for the clinician. Journal of Psychiatric Research, 12, 189-198.

Gedye, A. (1995) Dementia Scale for Down Syndrome. Gedye Research \& Consulting. http: / /www.gedye.ca

Mann, D. M. A. (1988) Alzheimer's disease and Down's syndrome. Histopathology, 13, 125-137.

Prasher, V. P. (1995) Age-specific prevalence, thyroid dysfunction and depressive symptomatology in adults with Down's syndrome and dementia. International Journal of Geriatric Psychiatry, 10, 25-31.

Streiner, D. L. \& Norman, G. R. (1999) Health Measurement Scales: A Practical Guide to Their Development and Use. Oxford University Press.

World Health Organization (1992) The ICD-I0 Classification of Mental and Behavioural Disorders: Clinical Descriptions and Diagnostic Guidelines. WHO. 